\title{
Las familias y la pandemia de la COVID-19
}

\author{
The families and the COVID-19 pandemic
}

As familias e pandemia COVID-19

\author{
Luis Diego Conejo \\ Universidad Nacional \\ ROR:.: https://ror.org/01t466c14 \\ Centro de Investigación y Docencia en Educación \\ Instituto de Estudios Interdisciplinarios de la Niñez y la Adolescencia \\ Heredia, Costa Rica \\ diego.conejo.bolanos@una.ac.cr \\ https://orcid.org/0000-0002-6415-0337 \\ Pablo Chaverri-Chaves \\ Universidad Nacional \\ ROR:: https://ror.org/01t466c14 \\ Centro de Investigación y Docencia en Educación \\ Instituto de Estudios Interdisciplinarios de la Niñez y la Adolescencia \\ Heredia, Costa Rica \\ pablo.chaverri.chaves@una.ac.cr \\ https://orcid.org/0000-0002-2639-4242 \\ Sara León-González \\ Universidad Nacional \\ ROR:: https://ror.org/01t466c14 \\ Centro de Investigación y Docencia en Educación \\ Instituto de Estudios Interdisciplinarios de la Niñez y la Adolescencia \\ Heredia, Costa Rica \\ sara.leon.gonzalez@una.ac.cr \\ iD https://orcid.org/0000-0003-2417-6783
}

Resumen: Este artículo pretende acercarse a plantear algunos elementos relevantes para responder a la siguiente pregunta: ¿cómo se ven afectadas las familias por la pandemia de la COVID-19? Primero se aborda la forma en que la pandemia ha obligado a las familias al confinamiento; luego se hipotetiza sobre la manera en que este confinamiento genera estrés y conflicto en las familias y se cierra con líneas de investigación y acción ante la nueva realidad.

Palabras claves: Familias; pandemia COVID-19; confinamiento; efectos sociales; efectos psicológicos. 
https://doi.org/10.15359/ree.24-S.10

https://www.revistas.una.ac.cr/index.php/educare

educare@una.ac.cr

\begin{abstract}
This article aims at closing the gap in our understanding in regards to the following question: how are families affected by the COVID-19 pandemic? First, it addresses how the pandemic has forced families into confinement; then it hypothesizes about how this confinement creates stress and conflict in families, and finally it addresses some lines of research and action regarding the new reality.
\end{abstract}

Keywords: Families; COVID-19 pandemic; confinement; social effects; psychological effects.

Resumo: Este artigo tem como objetivo chegar perto de levantar alguns elementos relevantes para responder à seguinte pergunta: como as famílias são afetadas pela pandemia COVID-19? Primeiro, aborda como a pandemia forçou as famílias a se confinarem; em seguida, fica hipotenso sobre como esse confinamento cria estresse e conflito nas famílias, e fecha com linhas de pesquisa e ação diante da nova realidade.

Palavras-chaves: Famílias; pandemia COVID-19; confinamento; efeitos sociais; efeitos psicológicos.

Recibido: 15/07/2020 Aceptado: 04/08/2020

\title{
Introducción
}

Las pandemias se caracterizan por brotes infecciosos a gran escala que aumentan en gran medida la morbilidad y la mortalidad en un área geográfica amplia y que además causan graves consecuencias a nivel económico, social y político (Madhav et al., 2017). La pandemia actual ha sido causada por la COVID-19, una enfermedad altamente infecciosa, que se contagia fácil y rápidamente de una persona a otras (Sanche et al., 2020) y para frenar su propagación, se han impuesto medidas de confinamiento que han implicado una restricción a la movilidad física y al contacto social de las personas, así como un freno a casi todas las actividades económicas, especialmente las que requieren presencialidad física. En este editorial, abordamos posibles efectos en las familias por esta pandemia en el contexto costarricense y algunas líneas de acción a tomar desde la academia.

El confinamiento trae una serie de consecuencias negativas para las personas, como, por ejemplo, respuestas ansiosas, sentirse de mal humor, tener pensamientos intrusivos sobre enfermedad, o preocupación excesiva y estrés por protegerse de la amenaza (Madani et al., 2020). La reacción adversa que experimentan las personas ante el confinamiento, se podría explicar por nuestro pasado evolutivo, como seres sociales que desarrollaron la capacidad de interconectarse con otras personas para crear entendimientos mutuos sobre el mundo y sobre sí mismos, siempre en relación con otros (Deci y Ryan, 2012; Tomasello et al., 2005).

Las familias, en tanto unidad social básica, constituyen lo que se ha llamado burbuja en términos epidemiológicos y que configura el espacio vincular primario en el que a las personas 
https://doi.org/10.15359/ree.24-S.10

se les ha solicitado confinarse para hacer frente a la pandemia. Por lo tanto, es en las familias donde se experimentan los efectos de la pandemia, más que a un nivel meramente individual. Es importante señalar que la pandemia de la COVID-19 no afecta a todas las familias ni a todos los sectores sociales de la misma forma. Aunque en un primer momento los contagios en Latinoamérica ocurrieron en personas que venían de Europa y Asia asociadas a condiciones socioeconómicas que les permiten hacer viajes internacionales, luego empezaron a darse con mayor frecuencia en personas y familias de condiciones socioeconómicas más bajas y de mayor vulnerabilidad. Es decir, se podría afirmar que, para el caso de Costa Rica, la ruta de contagio fue de los sectores de mayores ingresos hacia los de menores ingresos. Esto trae consecuencias importantes en cuanto al confinamiento para las familias, pues al inicio de la pandemia, las familias afectadas estaban caracterizadas en su mayoría por condiciones económicas favorables. Durante la segunda ola de contagios, las dificultades económicas de las familias afectadas (vivir en cuarterías, falta de acceso a servicios elementales de saneamiento, entre otros) han sido un obstáculo importante para apegarse al confinamiento.

\section{Líneas de investigación y acción que se deben priorizar}

Al presentarse cambios en las dinámicas familiares, los padres y las madres de familia se han visto en la obligación de aumentar la carga de sus roles parentales (por ejemplo, asumiendo roles protagónicos en el acompañamiento de los deberes escolares de sus hijos e hijas), incluso se puede señalar la pérdida de las redes de apoyo extendidas con las que contaban fuera de su burbuja social y que resultaban de soporte en muchas de estas situaciones. Como consecuencia, es importante investigar la afectación en su capacidad para dar respuesta oportuna a las necesidades e intereses de sus familias, lo cual podría agudizar situaciones de abuso, maltrato y violencia en los hogares. Las medidas de confinamiento disminuyen los niveles de actividad física, con lo cual se podría aumentar el sedentarismo y un uso indiscriminado y poco saludable de los dispositivos electrónicos, así como alteraciones en los patrones de sueño, horarios y rutinas diarias previamente establecidas. Estos efectos deben ser investigados y abordados para contribuir con la recuperación de las familias.

\section{Conclusión}

Los efectos de la pandemia en las familias no son homogéneos y se deben investigar y comprender a la luz de las necesidades familiares en sus contextos diversos actuales. Como un aporte para apoyar a las familias, desde el Instituto de Estudios Interdisciplinarios de la Niñez y la Adolescencia (INEINA) del Centro de Investigación y Docencia en Educación (CIDE), la respuesta ante esta situación ha consistido en la organización de videoconferencias sobre las relaciones familiares y la brecha educativa en tiempos de la COVID-19; así como la adaptación de cursos y atención a estudiantes, y la propuesta de proyectos con fondos concursables para acompañar 
https://doi.org/10.15359/ree.24-S.10

https://www.revistas.una.ac.cr/index.php/educare

educare@una.ac.cr

a las familias en la fase de atención y recuperación de la emergencia nacional por la COVID-19.

Nota general: Corrección de estilo y traducciones a cargo de las personas autoras.

\section{Referencias}

Deci, E. L. y Ryan, R. M. (2012). Self-determination theory. En P. A. M. Van Lange, A. W. Kruglanski y E. T. Higgins (Eds.), Handbook of theories of social psychology (Vol. 1, pp. 416-436). Sage. https:// doi.org/10.4135/9781446249215.n21

Madani, A., Boutebal, S. E. y Bryant, C. R. (2020). The psychological impact of confinement linked to the Coronavirus Epidemic COVID-19 in Algeria. International Journal of Environmental Research and Public Health, 17(10), 1-13. https://doi.org/10.3390/ijerph17103604

Madhav, N., Oppenheim, B., Gallivan, M., Mulembakani, P., Rubin, E. yWolfe, N. (2017). Pandemics: Risks, impacts, and mitigation. En D. T. Jamison, H. Gelband, S. Horton, P. Jha, R. Laxminarayan, C. N. Mock y R. Nugent (Eds.), Disease control priorities. Improving health and reducing poverty (Vol. 9, 3. ${ }^{a}$ ed., pp. 315-346). The World Bank. https://doi.org/10.1596/9781-4648-0527-1 ch17

Sanche, S., Lin, Y.T., Xu, C., Romero-Severson, E., Hengartner, N.y Ke, R. (2020). High contagiousness and rapid spread of severe acute respiratory Syndrome Coronavirus 2. Emerging Infectious Diseases, 26(7), 1470-1477. https://doi.org/10.3201/eid2607.200282

Tomasello, M., Carpenter, M., Call, J., Behne, T. y Moll, H. (2005). Understanding and sharing intentions: The origins of cultural cognition. Behavioral and Brain Sciences, 28(5), 675-691. https://doi.org/10.1017/S0140525X05000129 